\title{
FATORES DETERMINANTES DA LETALIDADE APÓS CIRURGIA PARA ENDOCARDITE INFECCIOSA
}

\author{
CHARLES ANDRE* ${ }^{*}$ MARCOS MARTINS DA SILVA**, \\ EDUARDO JORGE CUSTODIO DA SILVA** , MÁRCIA ARAÜJO SOUZA** , \\ EDUARDO SÉRGIO BASTOS ${ }^{* * *}$, SÉRGIO AUGUSTO PEREIRA NOVIS
}

\begin{abstract}
RESUMO - Estudamos a influência de dados demográficos, fatores predisponentes, aspectos clínicos, variáveis operatórias sobre a evolução em 39 pacientes operados por endocardite infecciosa (EI). Utilizamos os testes $t$ de Student, $\chi^{2}$ ou exato de Fisher em análises univariadas, regressão logistica para determinaçāo de fatores adversos independentes. $\mathrm{O}$ impacto do número destes fatores sobre a evoluçāo foi estudado pelo teste exato de Fisher. Valvas mais afetadas: aórtica (20) e mitral (16); germes mais comuns: Staphylococcus aureur (12) e Streptococcus $s p(10)$. Cirurgia de emergência e a presença de coma seis horas após a operação elevaram a letalidade $(p=0,001$ e $p=0,0015$ ), bem como infeç̧āo pelo S.uureus $(p=0,023)$ e presença de complicaçōes neurológicas ( $p=0,097$ ). A concomitância de dois ou três destes fatores elevou particularmente a letalidade (>76,9\%). Pacientes com EI devem receber cuidadosa avaliação quanto a indicação cirúrgica nas fases iniciais da doença, já que a concomitância de variáveis adversas e cirurgias em caráter de emergência elevam fortemente a letalidade por EI.
\end{abstract}

PALAVRAS-CHAVE: endocardite, letalidade hospitalar, complicações pós-operatórias.

\section{Postoperative mortality in infective endocarditis: determinant factors}

ABSTRACT - The factors leading to high postoperative mortality in active infectious endocarditis (IE) are poorly defined. We studied patients operated at an University Hospital between March 1978 and April 1992. We hipothesized that the summation of potential adverse factors would strongly increase mortality after surgery. We studied 39 patients ( 28 men), age range $13-70$ years (mean $\pm S D=32 \pm 16$ ) operated during active IE (time from onset $52 \pm 48$ days). Predisposing factor: rheumatic valvar disease in 14 cases, intravenous drug use in 5. Affected valves: aortic in 14, mitral in 10, tricuspid in 8, multiple structures in 7 . In most cases, $S$ aureus (12) or Streptoccocus sp (10) was isolated in blood cultures. Surgery was indicated in most patients because of heart failure (30), multiple embolic complications (17) or treatment failure (14). The possible adverse influence of specific demographic characteristics, clinical features and surgical variables was assessed by the Student $t$ test or the $\chi^{2}$ tcst. Also, multple regrcssion analysis was performed in order to identify independent adverse factors for increased mortality. Positive correlations were further investigated with the $\chi^{2}$ test to assess whether an increasing number of adverse factors could identify a special subset of patients with markedly elevated death risk. Fourteen patients (36\%) died after surgery. Emergency surgery $(p=0.001)$, the presence of coma 6 hours after surgery $(p=0.0015)$ and $S$. aureus infection $(p=0.023)$ were all associated with increased nortality. The presence of neurological complications was correlated with a high mortality (54\% vs. $27 \%)$. However this increase was of dubious statistical significance $(p=0.097)$. Multiple regression analysis confirmed $S$. aureus and emergency surgery as independent adverse factors for increased mortality. When put together, an increasing number of adverse factors was highly predictive of a fatal outcome, even

Hospital Universitário Clementino Fraga Filho (HUCFF) da Faculdade de Medicina (FM) da Universidade Federal do Rio de Janeiro (UFRJ): *Professor Assistente de Neurologia da FM/UFRJ; ** Professor Auxiliar de Neurologia da FM/UFRJ; ***Mestrando de Clínica Médica (Setor de Neurologia) da FM/UFRJ; ****Professor Adjunto de Cirurgia Cardíaca da FM/UFRJ; ******Professor Titular de Neurologia da FM/UFRJ. Trabalho apresentado no XIV Congresso Brasileiro de Neurologia - Porto Alegre - RS em 1992 e no Third International Conference on Stroke: Heart and Brain (Praga, República Tcheca, outubro-1995). Aceite: 21 -fevereiro-1997. 
after exclusion of that cvaluated after surgery (level of consciousness). Patients with two or three adverse factors had a very high mortality rate $(>76.9 \%)$. Mortality following surgery for active IE is increased in patients operated on an emergency basis especially if the infection is caused by $S$. cureus. The presence of neurological complications may also be associated with worse outcome. Early consideration of surgery should reduce the high mortality in patients with active IE.

KEY WORDS: endocarditis, hospital mortality, postoperative complications.

A endocardite infecciosa (EI) é uma das afecçōes cardiológicas com maior taxa de letalidade. Esta taxa varia $\mathrm{em}$ diferentes séries de 10 a $40 \%$, conforme o agente etiológico envolvido ${ }^{5}$. A maior parte dos pacientes é tratada com quimioterapia antimicrobiana. Entre os pacientes com alto risco, porém, há os que necessitam de intervenção cirúrgica ${ }^{6}$.

Os autores analisaram os prontuários dos pacientes operados no HUCFF/UFRJ em virtude de EI entre 1978 e 1992, com o objetivo de determinar os fatores demográficos, clínicos e operatórios preditivos de mortc após a operação.

\section{PACIENTES E MÉTODOS}

A análise de todos os casos de cirurgia valvar no período de março-1978 a abril-1992 revelou que 39 pacientes foram operados no curso de sua hospitalização inicial por EI, durante ou após o término da antibioticoterapia específica.

Foram analisados 28 homens e 11 mulheres, operados com idade entre 13 e 70 anos $(32 \pm 16)$. O intervalo médio entre o início da doença e o ato operatório foi de $52 \pm 48$ dias. As condiçōes predisponentes ao desenvolvimento da EI, valvas afetadas, germes causadores e indicações primárias para a intervenção cirúrgica são apresentadas na Tabela 1.

Previamente à cirurgia, ocorreram complicações neurológicas em 16 casos: alteração do nível de consciência, 8; hemiparesia, 6; síndrome meningea, 5; convulsões, 3; defeito de campo visual, 1.

O procedimento cirúrgico consistiu habitualmente de troca da valva acometida por prótese mecânica cm 2/3 dos casos. Próteses biológicas e tricuspidectomia foram utilizadas nos casos restantes. Foram utilizados a circulação extra-corpórea, pinçanento aórtico, hipotermia moderada $\left(28^{\circ} \mathrm{C}\right)$, e solução cardioplégica cristalóide gelada. Na anestesia foram utilizados fentanil, óxido nitroso e oxigênio.

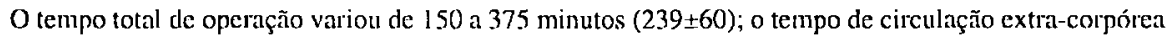
de 30 a 135 minutos (86 \pm 37 ). Durante o período perioperatório ocorreram complicaçōes em 16 casos: arritınias ventriculares, 6; hemorragia necessitando transfusão, 6; choque, 4; falência de desfibrilação, 1; e parada cardiorrespiratórii pré-operatórial, 1.

Tabela I. Caraterísticas clinicas $(n=39)^{l}$.

\begin{tabular}{|c|c|c|}
\hline Fator predisponente & Indicação cirúrgica? ${ }^{2}$ & Agente causal \\
\hline Febie reumática 14 & falência cardíaca 30 & S. aureus 12 \\
\hline Abuso de drogas 5 & embolização múltipla 17 & Streptococcus sp 10 \\
\hline Próterse valvar 2 & falência terapêutica 14 & S. epidermidis 2 \\
\hline Valva aórticil bicúspide 3 & insuficiência valvar aguda 4 & desconhecido 415 \\
\hline Outros 3 & distúrbio de condução 4 & \\
\hline \multirow[t]{2}{*}{ Descoshecido 12} & abscesso perivalvar 3 & \\
\hline & disfunção protética 2 & \\
\hline
\end{tabular}

1, a soma dass indicą̧ões cirúrgicas supera $39 \mathrm{~cm}$ funçăo da concomitância das mesmas em diversos pacientes; 2, cirurgial emergencial: 1.5 cisos; 3. un ciso associado a pseudomonas sp; 4, tratamento prévio à internilģio eın todas os cassos. 
Tabela 2. Letalidade pois-operatoria em endocardise infeccioss $(n=39)$.

\begin{tabular}{lcl}
\hline Fator estudado & $\begin{array}{c}\text { Letalidade } \\
\%(\mathrm{n})\end{array}$ & $\mathrm{p}$ \\
\hline cirurgia emergencial * & $67 \%(10 / 15)$ & 0,001 \\
coma 6h após cirurgia & $86 \%(6 / 7)$ & 0,0015 \\
infecção por S. aureus & $62 \%(8 / 13)$ & 0,023 \\
complicação neurológica & $54 \%(7 / 13)^{* *}$ & 0,097 \\
Total & $36 \%(14 / 39)$ & \\
\hline
\end{tabular}

* 15 casos - complicações presentes: falência cardíuca (14); embolizaç̧̄̃o múltipla (8); falência terapêutica (6); distúrbios de conduçiōo (2) **A Ateraçĩo do nível de consciência (8); heniparesial (5); irritaçĩo meníngea (4); convulsōes (3); outros (2); 3 pacientes exclúddos por doença neuropsiquiátrica prévia.

Tabela 3. Regressão logística: letalidade prevista como funs:äo de infecşäo pelo $\mathrm{S}$. aureus e cirurgia emergencial.

\begin{tabular}{lcc}
\hline Variável & Letalidade & $\begin{array}{c}\text { Razão das chances } \\
(95 \% \mathrm{CI})\end{array}$ \\
\hline $\begin{array}{l}\text { Cirurgia } \\
\text { emergencial' }\end{array}$ & $52 \%$ & $12,8(2,0-79,5)$ \\
$\begin{array}{l}\text { Infecção } \\
\text { por S. iureus }\end{array}$ & $38 \%$ & $7,3(1,1-47,5)$ \\
Ambos & $89 \%$ & \\
\hline
\end{tabular}

1) $\mathrm{P}=0,001$; 2) $\mathrm{P}=0,02$.
Faleceram após a operação I 4 pacientes (36\%). As causas cspecíficas de moite nesses casos foram: choque cardiogênico, 7; arritmia ventricular refratária, 2; complicaçōes septicêmicas, 2; lesão neurológica, 2; tamponamento cardíaco, 1.

Foi estudada a possível influência de 13 variáveis sobre a letalidade: idade, sexo; condição predisponente, valva afetada, germe; duração da doença até a operaçāo; indicaçāo cirúrgica, cirurgia de emergência, morbidade neurológica; tempo de cirurgia, tempo de circulação extra-corpórea, intercorrências perioperatórias; nivel de consciência no pós-operatório (6 horas).

Foram utilizados o teste $t$ de Student para amostras independentes no estudo das variáveis contínuas (idade, duração da doença, tempo de cirurgia e de circulação ex tra-corpórea); e o teste do $\chi^{2}$ nos casos restantes (teste exato de Fishcr quando o $\chi^{2}$ não pôde ser aplicado). As variáveis que exibiram correlação com maior letalidade pelos métodos mencionados acima foram adicionalmente estudadas mediante análise de rcgressão logística (SAS)" para identificação de fatores adversos independentes determinantes da letalidade. Buscamos ainda determinar a possível influência do número de fatores adversos sobre a evolução. Tal influência foi analisada pelo teste exato de Fisher em tabela $4 \times 2$ (SAS)". Além disto, investigamos a eventual existência de um ponto de corte nos valores desta variável. A hipótese testada pode ser assim formulada:

"A letalidade aprós operação pos endocardite infecciosa é proporcional ao nimero de fatores prognósticos adversos".

Foram considerados estatisticamente significativos os valores de $\mathrm{p} \leq 0,05$.

\section{RESULTADOS}

A Tabela 2 revcla as variáveis que sc associaram significativamente ao aumento da letalidade à análise univariada. A natureza emergencial da operação (principalmente por falência cardíaca) c a presença do estado de coma 6 horas após seu término revelaram-se preditores particularmente valiosos de evolução desfavorável, seguidos pela detecção de Staphylococcus auretus como germe causal. Embora não significativa, observa-se tendência de associação entre a presença de complicações neurológicas e a letalidade (duplicação da letalidade: $54 \%$ versus $27 \%$ ).

O estudo por regressão logística das três variáveis pré-operatórias detectadas à análise univariada (cirurgia emergencial, infecção pelo $S$. aureus. e complicações neurológicas) confirmou as duas primeiras como fatores adversos independentes determinantes da letalidade pós-operatória (Tabcla 3). Pacicntes com EI por $S$. aureus submetidos a cirurgia em carńter emergencial faleceram em $89 \%$ dos casos.

O tempo médio total de cirurgia foi discretamente maior $\mathrm{cm}$ casos de óbito $(227,8 \pm 65,1$ versus $258,4 \pm 43,7 ; p=0,1251)$ e o tempo de circulação extra-corpórca discrctamente inferior $(84,6 \pm 39,2$ versus $89,7 \pm 32,8 ; p=0,6816)$. Essas diferenças se mostraram, porém. insignificantes. $O$ 
Tabela 4. Influência do mímew de fatores* de risco sobre o risco de vida no pós-roperanirio de purcientes com endocardite infeciciost $(n=39)$.

\begin{tabular}{|c|c|c|c|c|}
\hline Número de falores & Óbito & Sobreviventes & Valor de & \\
\hline 0 & 1 & 97 & & \\
\hline 1 & 1 & & & \\
\hline 2 & 7 & $4, J$ & 0,003 & 0.007 \\
\hline 3 & 5 & $1\}$ & 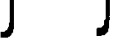 & \\
\hline
\end{tabular}

*4 fatores estudados: cirurgja emergencial, $S$. aureus como agente causal, morbidade neurológica no pré-operatório, coma no pós-operatório; no entiuto nenlum paciente apresentava os 4 fatores simultancamente.

Tabela 5. Influência do nímero de fatores adversos pré-operatórios* sobre a letalidade pós-opesatória na endocardite infecciosa $(n=39)$.

$\left.\begin{array}{ccccc}\hline \text { Número de fatores } & \text { Óbitos } & \text { Sobrevivência } & p & \text { cut-off }=2 \\ \hline 0 & 1 & 13 & & \\ 1 & 3 & 9 & 0,001 & \\ 2 & 8 & 2 & & \} 0,0002 \\ 3 & 2 & 1 & \end{array}\right\}$

*3 fatores selecionados por análise univaujada: cjrurgia de emergência, S. Aureus como agente causall, morbidade neurológica préoperatórial.

único paciente com mais de 70 anos faleceu. Entretanto, apenas 1 de 3 pacientes entre 60 e 69 anos morreu e a média etária dos pacientes que faleceram ou não foi semelhante $(31 \pm 14,4$ versus $33 \pm 18,1$; $\mathrm{p}=0,5806)$.

Estudando colctivamente as quatro variáveis indicadas na Tabela 2, detectou-se grande influência do nuimero de variáveis presentes sobre a cvolução $(p=0,0009)$. A Tabela 4 revela um ponto de corte evidente a partir de dois fatores adversos, com aumento de 8 vezes na taxa de letalidade: morte em 12 dos 17 casos ( $71 \%$ ) versus 2 dos $22(9 \%)$.

Análise semelhante foi feita considerando-se apenas três variáveis presentes antes do ato cirúrgico (Tabela 5). Novamente, detectou-se forte influência do número de variáveis presentes $(\mathrm{p}=0,001)$. Em semelhança à análise anterior, os pacientes com dois ou mais fatores adversos cxibiram grande aumento (4 vezes) da letalidade (10 de 13 casos $(62 \%)$ versus 4 de $26(15 \%)$.

\section{DISCUSSÃO}

O presente estudo tem diversas limitações, pois trata-se de análise retrospectiva com número relativamente pequeno de pacientes. As conclusões devem, assim, ser confirmadas em trabalhos futuros. Em particular, os resultados negativos, como a associação dúbia entre complicações neurológicas e a letalidade pós-operatória, podem decorrer do número reduzido de casos.

O achado mais importante desse estudo é a deteç̧ão de forte relação entre o múmero de fatores adversos presentes e a evolução pós-operatória desfavorável. Isso foi demonstrado levandose em conta as quatro variáveis prognósticas detectadas (Tabela 4) c, também, cstudando as três que puderam ser avaliadas antes do ato cirúrgico (Tabela 5). Obviamente, essa segunda análise é a mais importante do ponto vista prático, pois interessa aos médicos assistentes e aos cirurgiões cardíacos poder determinar a letalidade dos pacientes antes de proceder à cirurgia. 


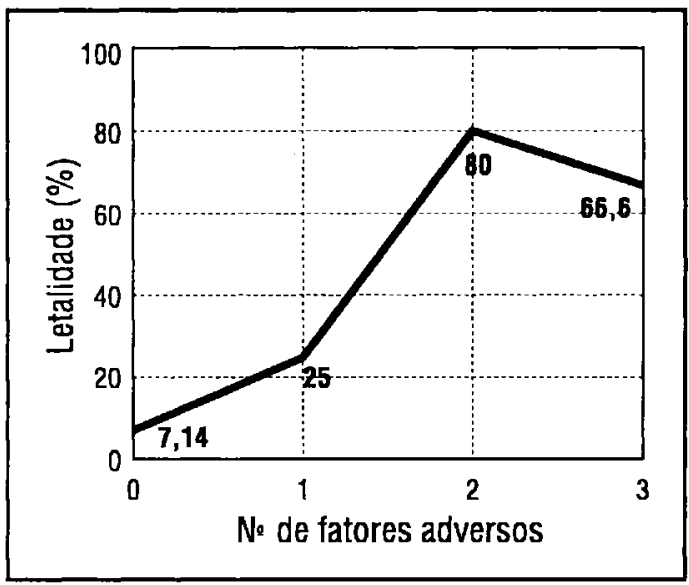

Fig 1. Letalidade por endocardite infecciosa em funçäo do nimero de fatores adversos. Observa-se a grande ascensāo da letalidade em pacientes com dosis ou mais farores adversos no periodo pré-operatório.

Além disso, pudemos demonstrar um ponto de corte nítido, que permite doravante quantificar, em un caso individual, a letalidade esperada após a cirurgia. É possível, assim, caracterizar um grupo de pacientes com alta letalidade, que exibem dois ou três dos seguintes fatores adversos: EI causada por $S$. aureus, cirurgia considerada emergencial, presença de complicações neurológicas. Por outro lado, pacientes que não exibem quaisquer desses fatores ou com apenas um deles exibem menor letalidade (Fig 1).

Nossos achados indicam grande aumento da letalidade nos pacientes operados em caráter de emergência, o que atesta a importância da indicação rápida e precisa deste procedimento na tentativa de evitar descompensaçāo clínica irreversível. Vários autores defendem a intervenção cirúrgica nas fases iniciais da doença ${ }^{1-3,5-7,10,11}$. Argumenta-se que, enquanto se espera a obtenção de um campo cirúrgico estéril, a lesão miocárdica em pacientes com disfunção hemodinâmica continua evoluindo e, frequentemente, associa-se a deterioração da funçāo renal ou neurológica. Estes fatores dificultariam a obtenção de bons resultados no pós-operatório ou estariam associados a sequelas graves ${ }^{7}$, de modo a transformar um procedimento eletivo com letalidade relativamente baixa cm intervençāo emergencial com alta letalidades.

Observamos nítida correlação prognóstica no que tange ao isolamento do Saureus como agente etiológico. Devemos destacar que em $75 \%$ dos pacientes que faleceram e apresentavam dois fatores adversos esta bacteria foi isolada. Isto parece indicar a importância do $S$. aureus no surgimento de outros fatores adversos como, por cxcmplo, morbidade ncurológica e acometimento cardiaco mais grave ${ }^{4.6 .8}$.

No presente estudo, o aumento da letalidade pós-operatória causado pela presença de complicaçōes neurológicas antes da cirurgia não alcançou significância estatística, enquanto variável isolada, talvez. em função do número reduzido de pacientes. Com efeito, Hannachi e col. ${ }^{\prime}$ citam como fator de pior prognóstico a existência de coma. Em contrapartida, Salgado e col ${ }^{\mathrm{h}}$ reportaram pouca relevância estatística ao correlacionar a presença de complicaçōes neurológicas e letalidade por EI. Nenhum dos dois autores faz menção específica aos pacientes que necessitavam de tratamento cirúrgico.

Em conclusão, nossos achados demonstram a importância prognóstica de fatores adversos tais como o caráter emergencial da cirurgia, o isolamento do $S$, aureus, talvez a presença de morbidade 
neurológica e, mais ainda, efeito sinérgico quando da concomitância de dois ou mais fatores. Assim sendo, a avaliaçāo cirúrgica nas fases iniciais da EI, sobretudo quando causada por $S$. aureus, pode permitir redução significativa da alta letalidade revelada neste e em outros estudos.

Agradecimentos - Somos gratos à Sr" Rosângela A. M. Noé, da Comissão de Investigação Científica HUCFF, pelo suporte estatístico a este estudo.

\section{REFERÊNCIAS}

1. Hannachi N, Béard T, Ismail MB. Les complications neurologiques des endocarditis infectieuses. Arch Mal Có $1991 ; 84: 81-86$.

2. Junatuinen MJ, Vãnttinen EA, Nikoskelainen J, Iinberg MV. Surgical treatment of native valve endocarditis. Scand $\mathbf{J}$ Thorac Cardiovasc Surg 1990;24:181-185.

3. Jubair KA, Fagh MRA, Ashneg A, Belhaj M, Sawyer W. Cardiac operations during active endocarditis. J Thorac Cardiovasc Surg 1992;104:487-490.

4. Kanter MC, Hart RG. Neurologic complications of endocarditis. neurology 1991;41:1015-1020.

5. Korzeniowski OM, Kaye D. Infective endocarditis. In Braunwald E (ed). Heart disease: a textbook of cardiovascular disease. Ed 4. Philadelfia: WB Saunders, 1992:1078-1105.

6. Larbalestier RI, Kinchla NM, Aranki SF, Couper GS, Collins JJ, Cohn LH. Acute bacterial endocurditis: optimising surgical results. Circulation 1992;86(Suppl. II):II-68-II-74.

7. Middlemost S, Wisenbaugh T, Meyerowitz C, Teeger S, Essop R, Skoularigis J, Cronje S, Sareli P, A case for early surgery in nutive left-sided endocarditis complicated by heart failure: results in 203 patients. J Am Coll Cardiol 1991; 18:663-667.

8. Silgado AV, Furlan AJ, Keys TF, Nichols TR, Beck GJ. Neurological complications of endocarditis: u I2-year experience. Neurology 1989;39:173-178.

9. Sus Institute Inc., SAS/STAT(18 User's Guide: version 6, Ed 4. Cary NC (USA). SAS Institute Inc., 1989 , Volume 2.

10. Varstela E, Verkkala K, Pohjola-Sintonen S, Valtonen V, Maamies T. Surgical treatment of infective alortic valve endocurditis. Scand J Thorac Curdiovasc Surg 1991;25:167-174.

11. Watunabe G, Haverich A, Speier R, Dresler C, Borst HG. Surgical treatment of active infective endocarditis with Paravalvular Involvement. J Thorac Cardiovasc Surg 1994;107:171-177. 